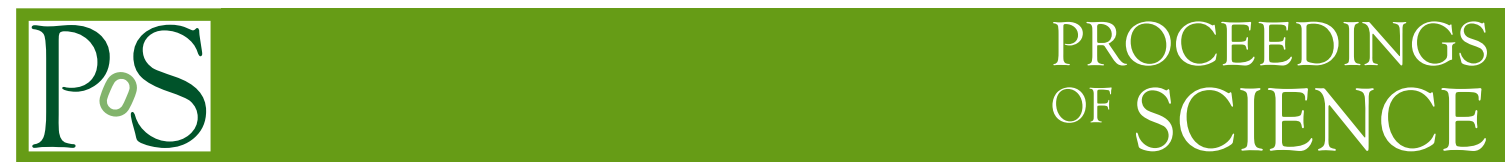

\title{
Status of the Muon g-2
}

\section{Simon Eidelman*}

Budker Institute of Nuclear Physics, Novosibirsk, Russia

E-mail: eidelman@inp.nsk.su

The current status of the Standard Model predictions for the muon anomalous magnetic moment is described. Various contributions expected in the Standard Model are discussed. After the reevaluation of the leading-order hadronic term based on the new $e^{+} e^{-}$data, the theoretical prediction is more than three standard deviations lower than the experimental value.

European Physical Society Europhysics Conference on High Energy Physics, EPS-HEP 2009, July 16 - 222009

Krakow, Poland

${ }^{*}$ Speaker. 


\section{Introduction}

For a particle with spin $\vec{s}$ and magnetic moment $\vec{\mu}$

$$
\vec{\mu}=g \frac{e}{2 m} \vec{s}
$$

where $e, m$ and $g$ are the charge, mass and gyromagnetic factor of the particle. In the Dirac theory of a charged pointlike spin-1/2 particle, $g=2$, and QED effects slightly increase the $g$ value. Conventionally, a quantity $a=(g-2) / 2$ is referred to as the anomalous magnetic moment.

The electron and muon anomalous magnetic moments have been measured with a very high relative accuracy of $0.24 \mathrm{ppb}$ [1] and $0.54 \mathrm{ppm}$ [2], respectively. The theoretical prediction for $a_{e}$ is only mildly affected by strong and weak interactions providing a test of QED and giving the most precise value of the fine-structure constant $\alpha$. In contrast, $a_{\mu}$ allows to test all sectors of the Standard Model since all of them contribute significantly to the total.

Although $a_{e}$ has been measured with much higher precision, $a_{\mu}$ is more sensitive to new physics effects: the gain is usually $\sim\left(m_{\mu} / m_{\mathrm{e}}\right)^{2} \approx 4.3 \cdot 10^{4}$. The $\tau$ lepton magnetic anomaly has even better potential, but because of the small lifetime of the $\tau$, it has not yet been measured, the best limits coming from DELPHI [3]: $-0.052<a_{\tau}<0.013$ at $95 \%$ confidence level. The sensitivity of the DELPHI measurement is still one order of magnitude worse than the predicted value of $a_{\tau}$ [4].

Any significant difference of $a_{\mu}^{\text {exp }}$ from $a_{\mu}^{\text {th }}$ indicates new physics beyond the Standard Model (SM). It is conventional to write $a_{\mu}$ as

$$
a_{\mu}^{\mathrm{SM}}=a_{\mu}^{\mathrm{QED}}+a_{\mu}^{\mathrm{EW}}+a_{\mu}^{\mathrm{had}},
$$

where the terms correspond to contributions of Quantum Electrodynamics (QED), electroweak (EW) and strong (hadronic) interactions. While discussing these terms and their precision, it is worth comparing them to the experimental result [2]:

$$
a_{\mu}^{\exp }=(11659208.0 \pm 6.3) \cdot 10^{-10} .
$$

The QED part is dominated by one graph, first-order in $\alpha$ [5]. The second- and third-order terms (up to $\alpha^{3}$ ) are known analytically [6]. Taking into account a recent more accurate numerical calculation of the $\alpha^{4}$ terms [7] and the leading $\log \alpha^{5}$ terms[8-10] one obtains

$$
a_{\mu}^{\mathrm{QED}}=(116584718.09 \pm 0.14 \pm 0.04) \cdot 10^{-11},
$$

where the errors are due to the uncertainties of the $\mathscr{O}\left(\alpha^{5}\right)$ term and $\alpha$, respectively, and the value of $\alpha^{-1}=137.035999084(51)$ from the latest measurement of $a_{e}$ has been used [1,11]. It is worth noting that the 4-loop term equals $38.1 \cdot 10^{-10}$ and is thus six times larger than the experimental uncertainty. Therefore, it is clear that its calculation as well as that of the 5-loop one is necessary.

The electroweak term is known rather accurately $[12,13]$ :

$$
a_{\mu}^{\mathrm{EW}}=(15.4 \pm 0.1 \pm 0.2) \cdot 10^{-10},
$$


where the first uncertainty is due to hadronic loops while the second one is caused by the errors of $M_{H}, M_{t}$ and 3-loop effects.

The hadronic contribution can also be written as a sum:

$$
a_{\mu}^{\mathrm{had}}=a_{\mu}^{\mathrm{had}, \mathrm{LO}}+a_{\mu}^{\mathrm{had}, \mathrm{HO}}+a_{\mu}^{\mathrm{had}, \mathrm{LBL}} .
$$

The dominant contribution comes from the leading-order term, which using dispersion relations can be written as $[14,15]$

$$
a_{\mu}^{\mathrm{had}, \mathrm{LO}}=\left(\frac{\alpha m_{\mu}}{3 \pi}\right)^{2} \int_{4 m_{\pi}^{2}}^{\infty} d s \frac{R(s) \hat{K}(s)}{s^{2}}
$$

where

$$
R(s)=\frac{\sigma\left(e^{+} e^{-} \rightarrow \text { hadrons }\right)}{\sigma\left(e^{+} e^{-} \rightarrow \mu^{+} \mu^{-}\right)}
$$

and the kernel $\hat{K}(s)$ grows from 0.63 at $s=4 m_{\pi}^{2}$ to 1 at $s \rightarrow \infty, 1 / s^{2}$ emphasizing the role of low energies. Numerically, $a_{\mu}^{\text {had,LO }} \approx 700 \times 10^{-10}$ [16], so we should know it to at least $1 \%$ to match the experimental accuracy.

\section{Evaluation of the hadronic term}

Several estimates of $a_{\mu}^{\text {had,LO }}$ appeared recently [17-19] based on the progress in the low energy $e^{+} e^{-}$annihilation and including the data not yet available previously [16,20,21].

One of the largest contributions to $a_{\mu}^{\text {had,LO }}$ comes from the $2 \pi$ final state (about $73 \%$ ) making high-precision measurements of the corresponding cross section mandatory. CMD-2 reported their results on the pion form factor $F_{\pi}$ from 370 to $1380 \mathrm{MeV}$ [22] with a systematic error of 0.6-0.8\% below $1 \mathrm{GeV}$, while SND measured $F_{\pi}$ from 390 to $970 \mathrm{MeV}$ with a systematic error of $1.3 \%$ [23]. KLOE studied $F_{\pi}$ using the method of radiative return or ISR [24,25] at $590<\sqrt{s}<970 \mathrm{MeV}$ with a sample of $3 \cdot 10^{6}$ events and systematic error of $0.9 \%$ [26]. The $\left|F_{\pi}\right|$ values from CMD2 and SND are in good agreement. The KLOE data are basically consistent with them with a somewhat different energy dependence. The contributions to $a_{\mu}$ from all three experiments agree. First results on the $2 \pi$ from BaBar show a slightly larger cross section [27]. Below $1.4 \mathrm{GeV}$ practically all final states have been measured with consistent results by the CMD-2 and SND groups in Novosibirsk [28]. Above $1 \mathrm{GeV}$, various final states with more than two hadrons were studied by BaBar [29] using the ISR method. They measure for the first time cross sections of a few new channels and also show that some of the previous results should be reconsidered. Using the new data below $1.8 \mathrm{GeV}$ in addition to the whole data set of $[16,20]$ for old experiments, one can reevaluate the leading-order hadronic contribution to $a_{\mu}$. In Table 1 we show the results for different energy ranges following [17].

The theoretical error consists of $1.9 \cdot 10^{-10}$ due to uncertainties of radiative corrections in old measurements and $0.7 \cdot 10^{-10}$ related to using pertubative QCD above $1.8 \mathrm{GeV}$. It can be seen that due to a higher accuracy of $e^{+} e^{-}$data the uncertainty of $a_{\mu}^{\text {had,LO }}$ is now much smaller than before [16,21].

The most recent estimate of the higher-order hadronic terms in [30] gives $a_{\mu}^{\text {had,HO }}=(-9.8 \pm$ $0.1) \cdot 10^{-10}$. The light-by-light hadronic contribution is estimated only theoretically [31]. Various predictions range between 80 and 136 (in units of $\cdot 10^{-11}$ ) with an uncertainty reaching $40 \cdot 10^{-11}$. 
Table 1

Updated $a_{\mu}^{\text {had,LO }}$

\begin{tabular}{cc}
\hline$\sqrt{s}, \mathrm{GeV}$ & $a_{\mu}^{\mathrm{had}, \mathrm{LO}}, 10^{-10}$ \\
\hline $2 \pi$ & $504.6 \pm 3.1 \pm 1.0$ \\
$\omega$ & $38.0 \pm 1.0 \pm 0.3$ \\
$\phi$ & $35.7 \pm 0.8 \pm 0.2$ \\
$0.6-1.8$ & $54.2 \pm 1.9 \pm 0.4$ \\
$1.8-5.0$ & $41.1 \pm 0.6 \pm 0.0$ \\
$J / \psi, \psi^{\prime}$ & $7.4 \pm 0.4 \pm 0.0$ \\
$>5.0$ & $9.9 \pm 0.2 \pm 0.0$ \\
\hline Total & $690.9 \pm 3.9_{\exp } \pm 2.0_{\mathrm{th}}$ \\
\hline
\end{tabular}

Using for the light-by-light term the result $(120 \pm 35) \cdot 10^{-11}$ and adding all hadronic contributions, we obtain $a_{\mu}^{\text {had }}=(693.1 \pm 5.6) \cdot 10^{-10}$. This result agrees with other estimations, e.g., [30, $32,18,19]$ and its accuracy benefits from the new $e^{+} e^{-}$data. Adding all theoretical contributions we obtain $a_{\mu}^{\text {th }}=(11659180.3 \pm 5.6) \cdot 10^{-10}$, i.e. $3.3 \sigma$ below the experimental value. Similar deviation is reported by Refs. [18,31].

How real is a very high accuracy of the leading-order hadronic contribution obtained above? To large extent it depends on our understanding of the radiative corrections due to initial-state radiation and vacuum polarization, and even more important effects of final state radiation. There is also a question of double counting of the hadronic final states in the leading- and higher-order hadronic terms [33], that of the missing states (e.g., final states with neutral particles only). One should also consider impact of the new ISR studies from BaBar, e.g., the contribution from the $K \bar{K} n \pi$ final states, previously estimated using isospin relations [20]. It is clear that new information may affect the size and accuracy of the continuum contribution below $2 \mathrm{GeV}$ (now $(62.4 \pm 2.0 \pm 0.5) \cdot 10^{-10}$ ) compared to that from the $\pi \pi$ (now $(504.6 \pm 3.1 \pm 1.0) \cdot 10^{-10}$ ).

There is still no explanation for the observed discrepancy between the predictions based on $\tau$ lepton and $e^{+} e^{-}$data [21]. The new high-statistics measurement of the two-pion spectral function by Belle [34] leads to about the same $a_{\mu}^{\text {had,LO }}$ as before despite some inconsistencies between Belle and ALEPH. On the other hand, a recent comprehensive analysis of the $e^{+} e^{-}$data below $1 \mathrm{GeV}$ and those on the $2 \pi$ decay of the $\tau$ lepton performed in Ref. [35] shows that two data sets can be reconciled if mixing between the $\rho, \omega, \phi$ mesons is taken into account in a consistent way. A recent reevaluation of isospin-breaking effects in [36] somewhat decreases the discrepancy, see Fig. 1.

What is the future of this SM test? From the experimental side there are suggestions to improve the accuracy by a factor of 2.5 at E969 (BNL) or even by an order of magnitude at JPARC. It is clear that it will be extremely difficult to improve significantly the existing accuracy of $a_{\mu}^{\text {had,LO }}$ by measuring the cross section of $e^{+} e^{-}$annihilation to better than $0.3 \%$ as required by future determinations of $a_{\mu}$ mentioned above. One can optimistically expect substantial progress 


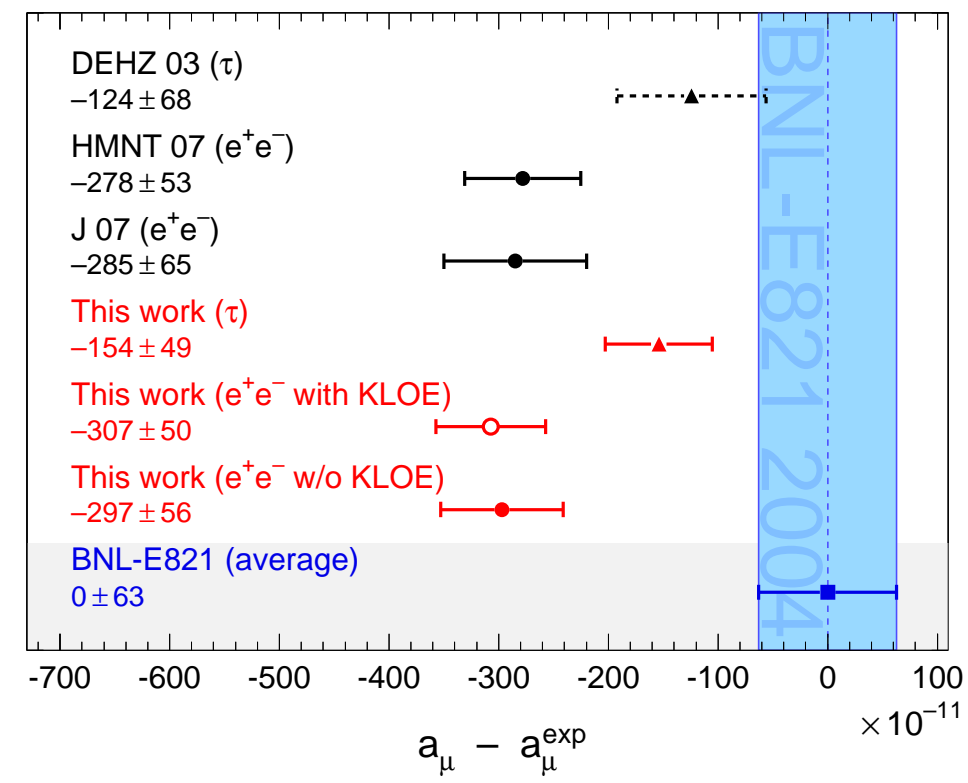

Figure 1: Comparison of $a_{\mu}$ from theory and experiment [36].

from new high-statistics ISR measurements at KLOE, BaBar and Belle together with the more precise determination of $R$ below 4-5 GeV from CLEO-c and BES-III. Experiments will start soon at VEPP-2000 now commissioning, which is a VEPP-2M upgrade up to $\sqrt{s}=2 \mathrm{GeV}$ with $L_{\max }=10^{32} \mathrm{~cm}^{-2} \mathrm{~s}^{-1}$ [37]. We can estimate that by 2010 the accuracy of $a_{\mu}^{\text {had,LO }}$ will be improved from $4.4 \cdot 10^{-10}$ by a factor of about 2 and the total error of $4.1 \cdot 10^{-10}$ will be limited by the LBL term $\left(3.5 \cdot 10^{-10}\right)$, still higher than the expected $2.5 \cdot 10^{-10}$ in E969.

Let us hope that progress of theory will allow a calculation of $a_{\mu}^{\text {had }}$ from first principles (QCD, Lattice). One can mention here a new approach in the QCD instanton model [38] or calculations on the lattice [39].

In conclusion, I'd like to emphasize once again that BNL success stimulated significant progress of $e^{+} e^{-}$experiments and related theory. Improvement of $e^{+} e^{-}$data led to substantial decrease of the $a_{\mu}^{\text {had,LO }}$ uncertainty. For the first time the accuracy of the theoretical prediction is better than that of the experimental measurement. Future experiments as well as development of theory should clarify whether the observed difference between $a_{\mu}^{\exp }$ and $a_{\mu}^{\text {th }}$ is real and what consequences for the Standard Model and for possible New Physics [40] it implies.

\section{Acknowledgments}

I'm indebted to M. Davier, F. Jegerlehner, M. Passera and G. Venanzoni for useful discussions and to my colleagues from VEPP-2M, CMD-2 and SND for the long-term collaboration.

This work was supported in part by the grants RFBR 07-02-00816, RFBR 08-02-13516, RFBR 08-02-91969, INTAS/05-1000008-8328, PST.CLG.980342 and DFG GZ RUS 113/769/0-2. 


\section{REFERENCES}

1. D. Hanneke et al., Phys. Rev. Lett. 100 (2008) 120801.

2. G.W. Bennett et al., Phys. Rev. D 73 (2006) 161802.

3. J. Abdallah et al., Eur. Phys. J. C 35 (2004) 159.

4. S. Eidelman and M. Passera, Mod. Phys. Lett. A 22 (2007) 159.

5. J.S. Schwinger, Phys. Rev. D 73 (1948) 416.

6. S. Laporta and E. Remiddi, Phys. Lett. B 379 (1996) 283.

7. T. Kinoshita and M. Nio, Phys. Rev. D 70 (2004) 113001.

8. A.L. Kataev, Phys. Rev. D 74 (2006) 073011.

9. P.A. Baikov, K.G. Chetyrkin, Ch. Sturm, Nucl. Phys. Proc. Suppl. 183 (2008) 8.

10. T. Aoyama et al., Phys. Rev. D 78 (2008) 113006.

11. M. Passera, Phys. Rev. D 75 (2007) 013002.

12. A. Czarnecki, B. Krause, W.J. Marciano, Phys. Rev. Lett. 76 (1996) 3267.

13. A. Czarnecki, W.J. Marciano, A. Vainshtein, Phys. Rev. D 67 (2003) 073006, Erratum-ibid. D 73 (2006) 119901.

14. C. Bouchiat and L. Michel, J. Phys. Radium 22 (1961) 121.

15. M. Gourdin and E. de Rafael, Nucl. Phys. B 10 (1969) 667.

16. S. Eidelman and F. Jegerlehner, Z. Phys. C 67 (1995) 585.

17. S. Eidelman, Proceedings of ICHEP-06, World Scientific, Vol.1, p.547, 2006.

18. K. Hagiwara et al., Phys. Lett. B 649 (2007) 173.

19. F. Jegerlehner, Nucl. Phys. Proc. Suppl. 181-182 (2008) 26.

20. M. Davier et al., Eur. Phys. J. C 27 (2003) 497.

21. M. Davier et al., Eur. Phys. J. C 31 (2003) 503.

22. R.R. Akhmetshin et al., Phys. Lett. B 648 (2007) 28.

23. M.N.Achasov et al., JETP 103 (2006) 380.

24. S. Binner, H.J. Kühn, K. Melnikov, Phys. Lett. B 459 (1999) 279.

25. M. Benayoun et al., Mod. Phys. Lett. A 14 (1999) 2605.

26. F. Ambrosino et al., Phys. Lett. B 670 (2009) 285.

27. B. Aubert et al., arXiv:0908.3589.

28. F.V. Ignatov, Nucl. Phys. Proc. Suppl. 181-182 (2008) 101.

29. B.Aubert et al., Phys. Rev. D 77 (2008) 092002.

30. K. Hagiwara et al., Phys. Rev. D 69 (2004) 093003.

31. F. Jegerlehner and A. Nyffeler, Phys. Rept. 477 (2009) 1.

32. J.F. Troconiz and F.J. Yndurain, Phys. Rev. D 71 (2005) 073008.

33. Yu.M. Bystritsky et al., JETP Lett. 83 (2006) 51.

34. M. Fujikawa et al., Phys. Rev. D 78, 072006 (2008).

35. M. Benayoun et al., Eur. Phys. J. C 55 (2008) 199.

36. M. Davier et al., arXiv:0906.5443.

37. S.I. Eidelman and S.I. Serednyakov, Nucl. Phys. Proc. Suppl. 131 (2004) 19.

38. A.E. Dorokhov, Nucl. Phys. A 790 (2007) 481.

39. T. Blum and S. Chowdhury, Nucl. Phys. Proc. Suppl. 189 (2009) 251.

40. D. Stöckinger, Nucl. Phys. Proc. Suppl. 181-182 (2008) 32. 